\title{
Role of epigenetic modifications in stem cell regulatory regions (Oct4, Sox2 and Nanog) and cancer
}

\author{
Mahira Arooj ${ }^{1}$, Faiza Abdul Rashid ${ }^{2}$, Asma Gul $^{2 *}$ \\ ${ }^{1}$ Department of Biochemistry, Quaid-i-Azam University Islamabad, Pakistan. \\ ${ }^{2}$ Department of Bioinformatics and Biotechnology, International Islamic University, Islamabad, Pakistan.
}

\begin{abstract}
Epigenetics is the study which involves the modifications in gene expression without changes in DNA nucleotide sequences. Methylation of DNA and chromatin remodeling through histone proteins are believed to be the most crucial epigenetic changes. Micro RNAs (miRNAs) are also shown to mediate the epigenetic gene regulation in mammals.DNA methylation can activate or inhibit the differentiation of stem cells by affecting the regulatory regions of its developmental genes like Oct4, Nanog and Sox2. In cancer, mutations in genetic as well as epigenetic elements play a vital role. The epigenetic changes in cancer are distinct in that they can be therapeutically reversed more easily. Aberrant DNA methylation (hypomethylation, hypermethylation) has its role at the molecular level in a number of cancers. This review will provide an insight into the epigenetic mechanisms involved in the regulation of stem cell specific genes Oct4, Sox2 and Nanog, alongwith the epigenetic modifications leading to cancer.
\end{abstract}

Keywords: Cancer epigenetics, DNA methylation, Epigenetics, Histone modifications, Oct4, Sox2 and Nanog, Stem cells.

\section{Introduction}

Somatic cells in an individual multicellular organism have basically identical genomes, but each of these cells has a distinct structure and function. This is due to the different uses of genes on the genome, that is, epigenetics [1]. Epigenetics is defined to consist of the changes that are heritable but without the involvement of DNA nucleotide sequences. It refers to the stably altered potential of gene expression, arising during development and proliferation [2]. Epigenome consist of three distinctive and entangled mechanisms which includes histone modifications, DNA methylation and post transcriptional gene regulation by non-coding microRNAs (miRNAs). The affect of these processes are chromatin compaction, transcript stability, nucleosome positioning, DNA folding and ample nuclear association of the heritable material [3].

Disease development can be certainly triggered by the disruption of the epigenome. Therefore complex interplay between genetic benefaction and epigenetic marks imprinted on a genome by endogenous and exogenous factors results in disease susceptibility [4]. Epigenetics might be a very promising and attractive avenue from a clinical point of view due to the reason that epigenetic alterations are potentially reversible unlike genetic variations (mutations, deletions, etc). Therefore unlike mutations, that exist whole life, restoration of epigenetically modified genes is possible for example, demethylation of methylated silenced genes can be acheived and histone complexes can be transcriptionaly activated by modification of acetylation and methylation of various histones via nutrients, drugs and other dietary interventions [5].

\section{Mechanisms of Epigenetics}

Epigenetic mechanisms are distinctive in nature and comprise histone modifications, DNA methylation and regulation by miRNAs. Diverse histone modifications may influence chromatin structure and direct methylation of DNA that may play an important role in tumorigenesis and gene regulation. The function of histone modification can include the regulation of significant cellular processes such asreplication, transcription and repair [9]. It can either activate or repress the gene depending on the residues involved and the type of modification existing, for example lysine acetylation results in transcriptional activation and its methylation results in transcriptional activation or repression considering the specific lysine modified [5]. Research has also been done in finding linkages between DNA methylation and histone modifications [10]. There may be various groups of enzymes that supplement or eliminate covalent modifications to histone proteins. Histone methyltransferases (HMTs) and histone acetyltransferases (HATs) supplement methyl and acetyl groups correspondingly, whereas histone demethylases (HDMs) and histone deacetylases (HDACs) remove methyl and acetyl groups correspondingly [5].

The major epigenetic adaptation in mammals is DNA methylation of cytosines at $\mathrm{CpG}$ dinucleotides [5] while it is revealed that methylation of cytosines that precede a base other than $\mathrm{G}$ (particularly $\mathrm{CpA}$ ) occurs to a lesser extent [6]. By the addition of a methyl group at the C5 position of the pyrimidine ring, 5Methylcytosine has been found to be distinguished from cytosine. The enzyme S-adenosyl-methionine catalyzes 
this reaction as a methyl group donor and also DNA methyltransferases (DNMT) play role in it [7]. DNA methylation, in alliance with histone adaptation is an indispensable constituent of the epigenetic mechanism, which controls gene expression and chromatin building. Throughout the human genome, $\mathrm{CpG}$ dinucleotides are not homogeneously distributed, but are frequently found in the promoter areas of genes, as well as in the large repetitive sequences like centromeric repeats, Long Interspersed Nuclear Element (LINE) and ALU retrotransposon components [8]. "CpG islands" have also been denoted to short CpG-rich areas, which are present additionally in $50 \%$ of human gene promoters $[5,7]$.

Greater part of the $\mathrm{CpG}$ dinucleotides, that are not linked with $\mathrm{CpG}$ islands are found chiefly methylated, in the mass of the genome. In disparity, the cytosines surrounded by CpG islands, particularly those coupled with promoter areas, are generally unmethylated. It has been declared that, if the suitable transcription factors are available, and the chromatin configuration permits contact to them, this deficit of methylation in promoter-associated $\mathrm{CpG}$ islands allows elucidation of the gene [11]. The exemption to this unmethylated status of $\mathrm{CpG}$ islands engross the silenced gene alleles for imprinted genes and genes include inside expanse of Xchromosome amelioration, and this point towards the stretched alliance of promoter DNA methylation and transcriptional silencing throughout ordinary mammalian maturity [7, 12].

Hypermethylation of $\mathrm{CpG}$ islands within gene promoters can silence gene, while promoters of actively transcribing genes stay hypomethylated [13]. By either preventing or promoting the recruitment of regulatory proteins to DNA, its methylation can silence the gene. For example it blocks access of transcription factors to binding sites (e.g. c-myc) and thus inhibits activation of transcription [14]. It can offer binding sites for methylbinding domain proteins in other instances, which can direct the gene repression by histone modifying enzymes $[5]$.

Presently, 40 imprinted genes have been reported to be regulated by DNA methylation. Even though, about 100 genes are in general predicted to be imprinted there appears to be more number of genes with epigenetic marks by DNA methylation [15].Demethylating agents such as 5-aza-20deoxycytidine (5-aza-CdR) and its homologues or valproic acid (VPA) have been found very precious for the analysis of DNA methylation [16].

Mirnas might be emerging as key mediators of epigenetic gene regulation in mammals besides DNA methylation and histone modifications. Expression of miRNA can be regulated by DNA methylation. Deregulation of miRNA recommends that its deregulation during carcinogenesis has an important suggestion for cancer regulation. Additionally, in tumour and normal cells the expression of miRNAs can be different [5].

\section{Components involved in epigenetic mechanisms}

The molecular mechanism of epigenetic gene silencing is apt to entail the synchronized accomplishments of DNA methyltransferases, HDACs, HMTs and supplementary chromatin modifying proteins, as a consequence (Figure 1) [17]. DNA methyltransferases (DNMTs), binding methyl-CpG-binding proteins (MBPs), HDAC, HATs, HMT, and Polycomb-group (PcG) proteins have been found to be involved in epigenetic ruling [18].

The alteration at 5-methylcytosine has been found to be impelled by diverse DNA methyltransferases (DNMTs). Three main DNMTs have been reported DNMT1 [N19], DNMT3a and DNMT3b [5]. MBP family of proteins has been found to be fit in to methyl- CpG-binding protein 2 (MeCP2) and methyl-CpG-binding domains 1-4 (MBD1, MBD2, MBD3, and MBD4) [3].Rest of the proteins (HATs, HDACs, and HMTs) have their role in chromatin remodeling [20].In neoplastic development, PcG proteins have been found to be the epigenetic chromatin modifiers. Two distinct multiprotein PcG complexes have been identified [21] namely polycomb repressive complex 1 (PRC1) and polycomb repressive complex 2 (PRC2) [18].

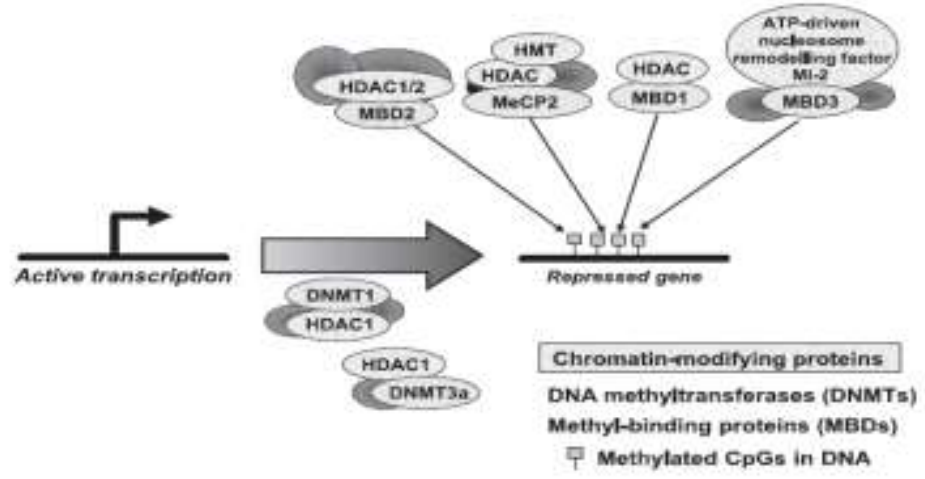

Figure.1. Epigenetic regulation of gene expression involves molecular links between DNA methylation and chromatin modifying proteins.

IV. DNA methylation and stem cells 


\subsection{Stem cells}

Stem cells play the main role in the progression and tissue homeostasis. The main characteristics ofstem cells are the self-renewal and the ability of differentiation into different lineages that are required for the tissue function and integrity [22].

\section{2. $\quad$ ESC and HESC}

Embryonic stem cells (ESCs) have been reported to be derived from the inner cell mass (ICM) of the early embryo and are capable to give rise to any cell kind (pluripotent cells) and in normal differentiate,during embryonic development, into three germ layers namely ectoderm, mesoderm and endoderm. On the other side, Adult stem cells have been studied as a small population of undifferentiated cells found in specific adult tissues andthey are lineage or tissue specific (multipotent). As an example, all blood lineages and mature blood elements are derived from hematopoietic stem cells (HSC) in bonemarrow. Adult stem cells have been found in many othertissues such as retina, liver, brain, epithelium, epiderm and follicule, nevertheless, it is very likely that stem cells arepresent in any tissue that go through renewal process[22].

ESCs have called as promising source of cells for regenerative treatment. Because of this, human ESCs (hESCs) have acknowledged substantial consideration ever since their beginning [23]. There have been found numerous sources of human stem cells with varying degrees of developmental strength. Immunosurgery has been used for the majority of reported hES derivations [24]. The main widespread technique for conventionally cultivated hES cells is vitrification of microdissected colonies in open or closed straws [25].

\subsection{Oct 4, Sox 2, Nanog in ESC and HESC}

The LIF and BMP signaling pathways have a central role in the preservation of a pluripotent stem cell phenotype. In addition these signaling pathways, transcription factors (TFs) are also vital for the specification of undifferentiated ES cells state [26].Oct4, Sox2 and Nanog are the core part of regulatory network, governing ESC pluripotency. They regulate common downstream genes that encourage pluripotency and self-renewal, whilst inhibiting differentiation processes [27].

Oct4 is found fundamental for pluripotency, self-renewal and embryogenesis[27].During the early stages of embryonic development,an essential role is played by Oct4 in establishing pluripotency [25].Oct4 is encoded by Pou5fl and is a POU homeodomain transcription factor that is expressed in the pluripotent cells of the ICM, epiblasts and primordial germ cells. Although Oct4 was reported to be a crucial factor in first differentiation, its overexprssion leads to pluripotency loss as cells differentiate into primitive endoderm and mesoderm lineages [28].

Nanog has the ability to maintain pluripotency in human and mouse ESCs, in the absence of feeder layer and LIF respectively [29,30].Nanog is expressed in the ICM and epiblast, and is down-regulated when cell differentiation occurs. Over-expression of Nanog in human ESCs leads to increased expression of primitive ectoderm markers [17].

Sox2 is another important pluripotency regulator in ESCs and its expression is not restricted to pluripotent cells, unlikeOct4 and Nanog.Sox 2 is detected in inner cell mass (ICM), epiblast and germ cells and also in primitive ectoderm and neural ectoderm[17]. As loss-of-function of Sox2 leads to defective epiblast and multiple lineage differentiation, therefore it is considered as an important pluripotency regulator and early cellfate [31].To regulate its own expression Sox2 complexes with Oct4 [32] and downstream mark genes such as FGF4[23].

Oct4, Sox2 and Nanog occupy about 353 genes in human embryonic stem cells. Up till now it is not clearly known that how these three stemness factors activate and repress the target genes but there is a possibility that they might be controlled by additional cofactors and by posttranslational modifications [27].

\subsection{DNA methylation in ESC and HESC}

In stem cells there are genomic regions that are methylated during the process of differentiation and are correlated with the gene repression. In most of the cells Oct4 $\mathrm{CpG}$ rich promoter is methylated leading to its repression, while in the ICM of blastocyst it is not methylated and hence expressed[33].It is suggested that changes in status of methylation and the chromatin structure occur before the lineage is specified [34]. Hence DNA methylation can activate or inhibit stem cell differentiation and is dependent on the cell type[16].

A number of developmental genes are silenced in ES cells by methylation of histone H3 lysine-4 and H3 lysine-27, while on the other hand the H3 lysine-4 methylation allows gene expression. Therefore these regions are called 'bivalent domains', as they carry both activating and repressing marks [35-37]. Due to these domains, the genes acquire the dual potential to become either actively transcribing or repressed depending on lineage specification. Current studies have shown that during tumourigenesis, these developmental genes show the tendency to acquire aberrant DNA methylation[38]. 


\section{Epigenetics of cancer}

\subsection{Cancer and its hallmarks}

It has been documented for many years that tumorigenesis is a multi-step procedure concerning manifold genetic modifications that donate to the continuing renovation of ordinary cells proceeding a malevolent phenotype [7].More lately, numerous features or 'trademarks' have been recognized that are common to all cancers. These consists of inattentiveness to anti-growth indicators, self-sufficiency in escalation signals, avoidance of apoptosis, immeasurable prospective for duplication, and possession of the mechanisms mandatory for tissue incursion, metastasis, and angiogenesis [39].

\subsection{Epigenetic mechanisms in cancer}

It is now well understood that cancer is contributed both by DNA mutations and epigenetic mechanisms [12]. There are few epigenetic mechanisms that are linked to cancer development, like DNA methylation ( hypermethylation or hypomethylation), loss of genetic imprinting (LOI) and histone modifications. DNA methylation and histone modifications are the main players of the three, when considering the epigenetics of cancer in mammals [40]. It has been anticipated that aberrant methylation of cytosine residues, inside these $\mathrm{CpG}$ islands, is the solitary most widespread abrasion in cancer cells even when evaluated to the overall rate of both mutations and cytogenetic deformitities. Aberrant methylation initiates at approximately $1.4 \%$ of $45,000 \mathrm{CpG}$ islands in the human genome, and may continue to as many as $10 \%$ of these islands during development of tumor [41]. A distinct feature of an epigenetic change in cancer is that it can be easily reversed by using therapeutic interventions while genetic change cannot be reversed [42]

Global DNA hypomethylation is found commonly in cancers and is mainly due to hypomethylation of repetitive sequences, even though there is mounting evidence for gene-specific modifications as well. A number of cancers, like thyroid, breast, cervical, prostate, stomach, lung, bladder, esophagus, colorectum and liver cancers, show global hypomethylation. Promoter hypomethylation is frequently correlated with the global hypomethylation. Hypomethylation of promoter $\mathrm{CpG}$ increases the expression of gene and also activates the proto-oncogenes [43]. Hypomethylation of repetitive DNA sequences and demethylation of intronic sequences, are found to be main causes of loss of methylation in cancer. The extent of genomic DNA hypomethylation increases during neoplasm development, as the lesion progresses from a benign proliferation of cells to metastatic cell [44].

It is the potential of global hypomethylation to contribute to a malignant phenotype by inducing effects on the chromosome functional stability, reactivation of transposable elements and loss of normal gene imprinting patterns [45]. Genome-wide demethylation further favors mitotic recombination with heterozygosity loss and chromosomal rearrangement promotion. In centromeric DNA, additionally, hypomethylation may promote aneuploidy in malignant cells [46, 44].

Intragenomic endoparasitic DNA can also be reactivated by DNA hypomethylation, such as L1 (long interspersed nuclear elements), and Alu (recombinogenic sequence) repeats. These elements can cause translocations when transcribed causing further genomic disruption. DNA methylation is also inadequately allied to DNA miss-match repair [44]. Besides global hypomethylation, the cancer cells genomes also show localized denovo hypermethylation, chiefly in $\mathrm{CpG}$ islands of tumour suppressor genes and microRNA (miRNA) genes. A major event in the cancer origin is the hypermethylation of promoter $\mathrm{CpGs}$ leading to inactivation of tumor suppressor genes. Hypermethylation of $\mathrm{CpG}$ islands within promoter regions of key tumour suppressor genes such as CDKN2A, MLH1, VHL and E-Cadherin shows correlation with their transcription repression in a few human cancers [44].

In this perspective, it has been suggested that, abnormal hypermethylation of promoter may put forth the same effects as do the mutation in coding region or deletion in one gene copy and therefore can be regarded as an additional pathway to contribute to the loss of one or both alleles [47, 7]. Hypermethylation of $\mathrm{CpG}$ islands can also silence the critical regulatory miRNAs in cancer [44].

\subsection{Cellular pathways and genes influenced by epigenetic alterations}

An epigenetic feature of cancer cells is the co-existence of gene-specific hypermethylation of promoter and global hypomethylation of genomic DNA. A number of these influenced genes have been recognized as tumor suppressor genes that are involved in regulation of cell cycle, DNA repair, apoptosis, angiogenesis, invasion, and achesion [48]. Studies have suggested that activated oncogenes, inactivated tumor suppressor genes, hormone receptors, cytokine receptors and growth factor receptors that contribute in these pathways, show regulation by epigenetic mechanisms. For example aberrant $\mathrm{CpG}$ methylation of $\mathrm{Rb}, \mathrm{p} 14, \mathrm{p} 15, \mathrm{p} 16$ and p73 disrupt the cell cycle control. Similarly CpG methylation in BRCA1 and hMLH1 leads to disruption of DNA damage repair system [18]. Numerous genes have been recognized in different cancers that show regulation by the epigenetic mechanisms. These genes are involved at diverse stages in the division, differentiation and cell 
proliferation. For example in breast cancer BRCA1, p16 and DAPK are epigenetically regulated and in leukemia p15, p73, MGMT, DAPK and CDH1 are regulated by epigenetic mechanisms [42].

\section{Conclusion}

In recent years the field of epigenetics has shown explosive growth. But the basic understanding is lacking, that how the major epigenetic marks function in the genome of eukaryotes. Only 100 or more known epigenetic marks have been studied for function. One of the most exhilarating area in epigenetic research is to understand the role of epigenetics in normal and cancer self-renewal. Epigenetic studies can be employed in the design of therapeutics, as currently being done in cancer therapy area. Genes that are essential for keeping the stem cells in pluripotent state, like Oct4, Nanog and Sox2, are also under the effect of epigenetic modifications. These developmental regulators normally remain silent in embryonic stem cells, but must preserve the potential to become active upon differentiation. Studies of epigenetics in the area of stem cells can be of great advantage and will provide insights into the regulatory mechanisms involved in differentiation and self renewal of stem cells.

\section{References}

[1] N. Mitsuyoshi, Epigenetics interaction of DNA methylation and chromatin, Gene, 278, 2001, 25-31.

[2] K. Shabana, A. Pranapda, K. Ikhlas, W. Larry and D. Asok, Epigenetic events associated with breast cancer and their prevention by dietary components targeting the epigenome, Chemical Research in Toxicology, 25, 2011, 61-73.

[3] S. Carla, V. Thomas, M. Rabih and H. Zdenko, Epigenetic drivers and genetic passengers on the road to cancer, Mutation Research, 642, 2008, 1-13.

[4] R. Jaenisch and A. Bird, Epigenetic regulation of gene expression: how the genome integrates intrinsic and environmental signals, Nat Genet, 33, 2003, 245-54.

[5] L. Alexander, B. Francesc and G. Ajay, Cancer chemoprevention by dietary polyphenols: Promising role for epigenetics, Biochemical Pharmacology, 80, 2010, 1771-1792.

[6] B.H. Ramsahoye, D. Biniszkiewicz, F. Lyko, V. Clark, A.P. Bird and R.Jaenisch, Non-CpG methylation is prevalent in embryonic stem cells and may be mediated by DNA methyltransferase 3a, Proceedings of the National Academy of Sciences, 97, 2000, 52375242 .

[7] G. Oliver, G. James, Herman and B.B. Stephen, The fundamental role of epigenetics, Blood Reviews, 20, $2006,1-13$.

[8] A. Bird, DNA methylation patterns and epigenetic memory, Genes and Development, 16(1), 2002, 6-21.

[9] T.Kouzarides, Chromatin modifications and their function, Cell, 128(4), 2007, 693-705.

[10] H. Martin and A. Marco, Epigenetics and human disease, The International Journal of Biochemistry \& Cell Biology, 41, 2009, 136146.

[11] Jones PA, Baylin SB. The fundamental role of epigenetic events in cancer. Nature Reviews Genetics. 2002;3:415-28.

[12] Staphen BB and james GH.DNA hypermethylation in tumorigenesis. Trends in Genetics. 2000;16( 4).

[13] Suzuki MM, Bird A. DNA methylation landscapes: provocative insights from epigenomics. Nature Reviews Genetics. 2008;9(6):465-76.

[14] Prendergast GC, Ziff EB. Methylation-sensitive sequence-specific DNA binding by the c-Myc basic region. Science. 1991;251(4990):186-9.

[15] Kunio S, Ryuzo Y. Epigenetics by DNA methylation for development of normaland cloned animals. Differentiation. 2002;69:162166.

[16] Tim C, Roloffab, Ulrike A. Nuber. Chromatin, epigenetics and stem cells.European Journal of Cell Biology. 2005;84:123-135.

[17] Jonathan G, MoggsJI, Goodman JE, Troskoand Ruth AR. Epigenetics and cancer implications for drug discovery. Toxicology and Applied Pharmacology. 2004;196:422- 430.

[18] Sonia MN and Manel E. Epigenetic biomarkers for human cancer: The time is now. Critical Reviews in Oncology/Hematology.2008; 68:1-11.

[19] Plass C. Cancer epigenomics. Human Molecular Genetics. 2002;11(20):2479-88.

[20] Fraga MF, Ballestar E, Villar-Garea A, et al. Loss of acetylation at Lys16 and trimethylation at Lys 20 of histone H4 is a common hallmark of human cancer. Nature and Genetics. 2005;37:391-400.

[21] Lund AH, van Lohuizen M. Polycomb complexes and silencing mechanisms. Current Opinion in Cell Biology. 2004;16:239-46.

[22] Vivek S, Thomas V, Zdenko H. Histone acetylation and chromatin signaturein stem cell identity and cancer. Mutation Research 2008;637:1-15.

[23] Silva J, Smith A. Capturing pluripotency. Cell. 2008;132:532-536.

[24] Chen X, Xu H, Yuan P, Fang F, Huss M, Vega VB, Wong E, Orlov YL, Zhang W, Jiang J, Loh YH, Yeo HC, Yeo ZX, Narang V, Govindarajan KR, Leong B, Shahab A, Ruan Y, Bourque G, Sung WK, Clarke ND, Wei CL, Ng HH. Integration of external signaling pathways with the core transcriptional network in embryonic stem cells. Cell. 2008;133:1106-1117.

[25] Nichols J, Zevnik B, Anastassiadis K, Niwa H, Klewe-Nebenius D, Chambers I, Scholer H, Smith A. Formation of pluripotent stem cells in the mammalian embryo depends on the POU transcription factor Oct4. Cell. 1998;95:379-391.

[26] Xi C et al. Integration of External SignalingPathways with the Core TranscriptionalNetwork in Embryonic Stem Cells. Cell. 2008;133:1106-1117.

[27] laurie et al. Core Transcriptional Regulatory Circuitry in Human Embryonic Stem Cells. Cell. 2005;1226:947-956

[28] Niwa H, Miyazaki J, Smith AG. Quantitative expression of Oct-3/4 defines differentiation, dedifferentiation or self-renewal of ES cells. Nature Genetics. 2000;24:372-376.

[29] Chambers I, Colby D, Robertson M, Nichols J, Lee S, Tweedie S, Smith A. Functional expression cloning of Nanog, a pluripotency sustaining factor in embryonic stem cells. Cell. 2003;113:643-655.

[30] Mitsui K, Tokuzawa Y, Itoh H, Segawa K, Murakami M, Takahashi K, Maruyama M, Maeda M, Yamanaka S. The homeoproteinNanog is required for maintenance of pluripotency in mouse epiblast and ES cells. Cell. $2003 ; 113$ (2003):631-642.

[31] Avilion AA, Nicolis SK, Pevny LH, Perez L, Vivian N, Lovell-Badge R, Multipotent cell lineages in early mouse development depend on SOX2 function. Genes Development. 2003;17:126-140.

[32] Chew JL, Loh YH, Zhang W, Chen X, Tam WL, Yeap LS, Li P, Ang YS, Lim B, Robson P, Ng HH. Reciprocal transcriptional regulation of Pou5f1 and Sox2 via the Oct4/Sox2 complex in embryonic stem cells. Molecular Cell Biology. 2005;25:6031-6046. 
[33] Deb-Rinker P, Ly D, Jezierski A, Sikorska M, Walker PR. Sequential DNA methylation of the Nanog and Oct-4 upstream regions in human NT2 cells during neuronal differentiation.Journal of Biological Chemistry.2004. E-pub

[34] Tagoh, H, Melnik S, Lefevre P, Chong S, Riggs AD, Bonifer C. Dynamic reorganization of chromatin structure and selective DNA demethylation prior to stable enhancer complex formation during differentiation of primary hematopoietic cells in vitro. Blood. 2004;103:2950-2955.

[35] Azuara V et al. Chromatin signatures of pluripotent cell lines. Nature Cell Biology. 2006;8:532-538.

[36] Bernstein BE et al. A bivalent chromatin structure marks key developmental genes in embryonic stem cells. Cell. 2006;125:315326.

[37] Mikkelsen TS et al. Genome-wide maps of chromatin state in pluripotent and lineage-committed cells. Nature. 2007;448:553-560.

[38] Mae P and Robert F. Epigenetic stability of embryonic stem cells and development potential. TRENDS in Biotechnology. 2007;25:12.

[39] Jonathan G, Moggs JI, Goodman, James E, Trosko and Ruth A and RobertsEpigenetics and cancer implications for drug discoveryand safety assessment. Toxicology and Applied Pharmacology. 2004;196:422-430.

[40] Q L et al. Epigenetics, disease, and therapeutic interventions. Ageing Research Reviews. 2006;5: 449-467.

[41] Dominique $\mathrm{Z}$ et al. The role of epigenetics in environmental and occupational carcinogenesis. Chemico-Biological Interactions. 2010;188:340-349.

[42] Mukesh V and Sudhir S. Epigenetics in cancer implications for early. Lancet Oncol.2002;3:755-63.

[43] Q L et al. Epigenetics, disease, and therapeutic interventions. Ageing Research Reviews. 2006;5: 449-467.

[44] Inhua MC and Renato P. Epigenetics unforeseen regulators in cancer. BiochimicaetBiophysicaActa. 2001;1552:15-26.

[45] Ehrlich M. DNA methylation in cancer: too much, but also too little. Oncogene .2002;21:5400-13.

[46] Gaudet F, Hodgson JG, Eden A, Jackson-Grusby L, Dausman JW, Gray JW, et al. Induction of tumors in mice by genomic hypomethylation. Science. 2003;300:489-92.

[47] Herman JG, Baylin SB. Gene silencing in cancer in association with promoter hypermethylation. The New England Journal of Medicine. 2003;349:2042-54.

[48] Thomas V, Carla S, Zdenko H. Epigenetic interplay between histone modifications and DNA methylation in Gene silencing. Mutation Research. 2008;659:40-48 